\title{
Real-Life Tolerability and Effectiveness of Adalimumab Biosimilar in Rheumatoid Arthritis: ASPIRE Registry Data
}

\author{
Sanjiv Kapoor • Viswanath V. Kaushik - Rahul Jain • Vijay Rao • \\ Mihir Gharia (D)
}

Received: May 10, 2019 / Published online: June 28, 2019

(C) The Author(s) 2019

\begin{abstract}
Introduction: The TNF- $\alpha$ blocker adalimumab is a well-proven therapy for rheumatoid arthritis (RA). A biosimilar adalimumab (ZRC-3197; Exemptia $^{\mathrm{TM}}$ ), a 'fingerprint match' to reference adalimumab, has been approved for prescription in India since 2014. Here, we report on the effectiveness and tolerability of this biosimilar adalimumab (bADA) from the Adalimumab Biosimilar Patient Registry [ASPIRE; ISRCTN16838474], which contains data from real-life RA patients from India.

Methods: ASPIRE is a post-marketing, observational registry that evaluates real-world experience across multiple centres in India. Patients
\end{abstract}

Enhanced Digital Features To view enhanced digital features for this article go to: https://doi.org/10.6084/ m9.figshare.8288894.

S. Kapoor

Indian Spinal Injuries Centre, New Delhi, India

V. V. Kaushik

Arthritis \& Rheumatism Centre, Chennai, India

R. Jain

Narayana Multispeciality Hospital, Jaipur, India

V. Rao

Rheumatology, Manipal Hospital, Bangalore, India

M. Gharia $(\bowtie)$

Medical Affairs, Zydus Biovation, Cadila Healthcare

Ltd, Ahmedabad, India

e-mail: mihir10584@gmail.com with moderate to severe RA who were prescribed bADA $40 \mathrm{mg}$ subcutaneously every fortnight were enrolled. Patients with complete data available until 24 weeks of bADA treatment were extracted and analyzed for standard disease activity measures and reported adverse events.

Results: The registry included 149 patients with RA who had a median age of 41 (22-67) years; $65 \%$ of the patients were female. Disease outcome measures, i.e. ESR, DAS-ESR and VASpain scores, showed gradual and significant decreases $(p<0.0001$ for all) in 73 analyzable patients who received 24 weeks of bADA therapy. ACR20, ACR50 and ACR70 responses were achieved in $48 \%, 48 \%$ and $34 \%$ of patients after 24 weeks of therapy, respectively, and about $58 \%$ and $15 \%$ of patients were moderate and good EULAR responders, respectively. Physician and patient ratings for the overall global assessment of efficacy and tolerability were 'good' to 'excellent' for the majority of the patients $(\geq 96 \%)$. No new safety signals were observed when analyzing this registry data.

Conclusion: Real-life data from this post-marketing observational analysis demonstrate the clinical effectiveness and tolerability of 24 weeks of adalimumab biosimilar therapy in Indian patients with RA. This report also reflects upon the treatment strategies and prescription patterns for such therapies in Indian clinical practice. 
Trial Registration: ISRCTN16838474.

Funding: Cadila Healthcare Limited, India.

Keywords: Adalimumab; Biosimilar; Real-life; Registry; Rheumatoid Arthritis

\section{INTRODUCTION}

Rheumatoid arthritis (RA) is a chronic, systemic, autoimmune disorder that is primarily characterized by synovial inflammation and joint destruction leading to functional loss, despite the availability of traditional therapies such as conventional synthetic disease-modifying antirheumatic drugs (csDMARDs). Over the past few decades, biologics have revolutionized the management of RA patients, particularly those with RA resistant to csDMARDs. The most promising agents of this type are the tumour necrosis factor$\alpha$ (TNF- $\alpha$ ) inhibitors [1, 2], such as adalimumab (Humira ${ }^{\circledR}$; Abbott, USA) which is a fully humanized immunoglobulin (IgG1) monoclonal antibody being successfully used to treat moderate to severe RA since 2002 [2]. Sufficient evidence is already available for its efficacy and safety in reducing RA activity, improving quality of life and preventing structural damage in RA patients [3-6]. However, the accessibility and affordability of biologic therapies are always a concern in less resourceful regions of the world, leading to limited experience in the clinical use of biologics in such areas. This is of particular concern in countries with developing economies such as India, where treatment costs are mostly borne by the patients, which also impacts the prescription patterns and treatment approaches employed by the physicians.

With reference biologics coming off-patent, affordable biosimilar versions of those biologics have entered the market after having passed though rigorous comparative evaluation pathways [7-9]. ZRC-3197 (Exemptia ${ }^{\text {TM} ; ~ C a d i l a ~}$ Healthcare Ltd, India); is one such adalimumab biosimilar that has been approved for use in India since 2014 [10, 11]. The physicochemical, functional and clinical comparability of this adalimumab biosimilar (bADA) to reference adalimumab has been demonstrated in detail via standard techniques and clinical trial
$[12,13]$. Based on those initial results, ZRC-3197 was authorized for clinical use in India. Recently, clinical reports from real-world settings have described the effectiveness and tolerability of this bADA in Indian patients with various immune-mediated chronic inflammatory disorders [14-16].

Other adalimumab biosimilars apart from Exemptia $^{\mathrm{TM}}$ are also available in the Indian market. Each of these biosimilars has its own distinct properties, so it is important to monitor the performance of biosimilars during the post-approval phase in real-world clinical practice [11]. More importantly, in countries such as India, the prescription patterns and durations of biologic treatments are influenced more by the patient's relief from symptoms and their ability to pay than the perception of disease severity or outcomes. Hence, the generation of real-world data that allow us to probe these practical patient-centric nuances and outcomes is of the utmost importance when managing these patients.

The Adalimumab Biosimilar Patient Registry [ASPIRE] represents one such attempt undertaken as part of the post-marketing surveillance to evaluate the usage and efficacy of the bADA in various autoimmune arthritic conditions in the real world. In this paper, we report our analysis of the registry's data on the effectiveness and tolerability of the bADA after 24 weeks of clinical use in real-world RA patients. We also reflect upon the treatment and usage patterns of this agent in Indian patients. Data for AS patients are also being reported separately.

\section{METHODS}

The Adalimumab Biosimilar Patient Registry (ASPIRE) is an ongoing, multicentre, non-interventional, open-label, observational datacollection registry (ISRCTN16838474) that was created to facilitate the evaluation of real-world patients with rheumatic disorders treated with the bADA Exemptia ${ }^{\mathrm{TM}}$. This registry was initiated by Cadila Healthcare Ltd in November 2015 across multiple centres in India as part of their postmarketing obligations to the regulatory agencies. Patients with moderate to severe active RA, ankylosing spondylitis, psoriatic 
arthritis and juvenile idiopathic arthritis treated with the bADA were included after voluntary consent. Ethics committee approval (Intersystem Biomedica Ethics Committee, 09/02/2018, ref. ISBEC/NR-3/DD-JJ/2018) was sought for data analysis and publication. Patient consent for participation was duly taken. This study was conducted in accordance with the 1964 Declaration of Helsinki and applicable amendments.

Real-world patients with moderate to severe RA who were eligible as per the ACR guidelines and had failed to respond or had responded inadequately to csDMARDs or other biologics were considered for bADA therapy. All patients tested negative for tuberculosis using the Mantoux test $(<5 \mathrm{~mm})$, gamma interferon and chest $\mathrm{X}$-ray; had normal blood counts and liver and renal function; and had elevated ESR and CRP levels. Although not an obvious exclusion criterion, patients were tested for hepatitis B and C and HIV, and seropositive patients were not considered for bADA therapy by physicians in India as per their routice clinical practice pattterns in order to avoid any additional risks to these patients. All patients had received standard csDMARD treatment for at least 3 months before the start of bADA therapy as per clinical practice. All these patients were treated with bADA $40 \mathrm{mg}$ subcutaneously every other week as per the centre's routine clinical practice. Most of the patients received stable doses of methotrexate concomitantly at the treating physician's discretion.

Data management was performed by an independent clinical research organization. Electronic case report forms were set up and data were entered for all enrolled patients by all participating centres at baseline and routine follow-up visits. Patient data obtained during routine follow-up visits and investigations performed up to 24-28 weeks after therapy initiation were collected until the data cutoff date of May 2017. Only evaluable patients with complete data were then analyzed. We did not attempt to correct for missing data. Patient demographics (age, sex, race, etc.) and clinical characteristics (disease activity, history of comorbidities and treatment history) were recorded at the time of initiation of bADA therapy. Key efficacy outcomes evaluated for RA patients included American College of Rheumatology (ACR) improvement criteria, i.e. ACR20, ACR50 and ACR70; Disease Activity Score-28 for Rheumatoid Arthritis with ESR (DAS28-ESR), European League Against Rheumatism (EULAR) response criteria, visual analogue scale (VAS) for pain, and overall global efficacy assessment by physician and patient using a 4-point Likert Scale. Safety and tolerability were concluded based on adverse events as reported by the physician or patient. The global tolerability assessment ratings by physician and patient were also based on a 4-point Likert Scale, as follows: excellent (if no adverse event); good (mild adverse event); fair (more than two mild or one moderate adverse event); or poor (serious adverse event requiring hospitalization).

Data were analyzed independently with SAS version 9.4 or later. Descriptive statistics such as $n$, mean, median, standard deviation, range (minimum, maximum) and percentage change were used to summarize continuous variables. Frequencies and percentages were computed for categorical data. Statistical analyses were performed using the Wilcoxon signed rank test to compare efficacy variables at a significance level of $5 \%$.

\section{RESULTS}

As of May 2017, 502 patients with autoimmune inflammatory conditions were included in the ASPIRE registry. From this, data on 149 RA patients were retrieved for analysis. Data collection was attempted for all parameters for the 149 patients who started to receive bADA therapy, but only at their regular clinic visits as per their feasibility. Some patients were either discontinued from the therapy at the physician's discretion, missed a clinic visit, had a delayed follow-up schedule that did not match the required timepoint for data collection, or did not undergo all the clinical and diagnostic tests required for outcome evaluations, as anticipated with routine clinical care and centre-tocentre variation in data collection. Hence, data for all parameters were not available for every patient at the 6-month follow-up visit. Thus, for 
the efficacy outcome analysis, the complete data available for 73 patients were considered (i.e. we focussed on patients for whom complete data for weeks 24-28 post adalimumab biosimilar therapy were available). Overall global efficacy and safety assessment results were available for 126 patients at the end of 24 weeks, and are reported here.

The demographic and clinical characteristics for all of the RA patients included in the registry are presented in Table 1 to provide an overall patient profile. The median age for the RA group was 41 (22-67) years and $65 \%$ of that group were females. The duration of disease was $>6$ years. Before the initiation of bADA treatment, the majority of the patients (87\%) had received prior csDMARDs, mostly methotrexate $(78 \%)$; about $8 \%$ of the patients had received biologics such as infliximab and etanercept; and $40 \%$ of the patients were on NSAIDs. Comorbid conditions included vitiligo

Table 1 Baseline demographic and clinical characteristics of the RA patients

\begin{tabular}{|c|c|}
\hline Patient characteristic & $\mathrm{RA}(N=149)$ \\
\hline Male:female, $n(\%)$ & $35: 65$ \\
\hline Age (years) & $42.1 \pm 9.5$ \\
\hline $\operatorname{BMI}\left(\mathrm{kg} / \mathrm{m}^{2}\right)$ & $26.0 \pm 3.5$ \\
\hline CRP & $26.7 \pm 23.4$ \\
\hline Duration of disease & $6.4 \pm 5.4$ \\
\hline VAS-pain & $8.5 \pm 0.8$ \\
\hline DAS28-ESR & $7.1 \pm 0.4$ \\
\hline Prior csDMARDs [yes], $n(\%)$ & $129(86.6 \%)$ \\
\hline Methotrexate & $116(77.8 \%)$ \\
\hline Hydroxychloroquine & $3(2.0 \%)$ \\
\hline Leflunomide & $1(0.7 \%)$ \\
\hline Sulfasalazine & $1(0.7 \%)$ \\
\hline Others & $8(5.3 \%)$ \\
\hline Prior biologics [yes], $n(\%)$ & $12(8 \%)$ \\
\hline Nonsteroidal anti-inflammatory drugs & $60(40.2 \%)$ \\
\hline
\end{tabular}

Data presented as mean \pm standard deviation or number of patients (percentage) or hypertension (in $0.5 \%$ each), obesity and coronary artery disease (in $2 \%$ ), and diabetes (in $1.5 \%$ of these patients). About $70 \%$ of the patients continued to receive methotrexate concomitantly with bADA therapy. The baseline DAS28-ESR score was 7.16 (4.7-7.7) and the baseline VAS-pain scale score was 9.0 (5-10) for the group.

The disease outcome measures evaluated, i.e. ESR, single joint count (SJC), total joint count (TJC), patient global assessment (PGA), DAS-ESR and VAS-pain scores, showed gradual decreases over the treatment period of 24 weeks for the 73 patients with complete data. The improvements in these scores after 24 weeks of bADA therapy are presented in Table 2. There were significant decreases $(p<0.0001$ for all) in the ESR, DAS28-ESR, and VAS-pain scores at 24 weeks as compared to the baseline. The mean change in DAS28-ESR post 24 weeks was $-3.53 \pm 0.68$, and the percentage change was $-48.6 \pm 7.58$.

At the 24-week follow-up, $48 \%$ of the patients achieved ACR20 and ACR50 responses and $34 \%$ of the patients achieved an ACR70 response. About $58 \%$ of the patients achieved a moderate response and $15 \%$ of the patients achieved a good response as per the EULAR criteria. The physician and patient global efficacy assessment ratings were 'good' to 'excellent' for the majority of the treated group ( $\geq 96 \%$ patients; $n=126$ ) (Table 3 ).

In general, the most commonly reported adverse events (in about 10\% of patients) were abdominal discomfort, abdominal pain, accelerated hypertension, arthralgia, body tinea, chest pain, diarrhoea, dizziness, dyspepsia, dyspnoea, fungal infection, gastritis and headache, which were in line with the approved prescribing information for adalimumab biosimilar and consistent with the safety profile of adalimumab. Infections were reported in $2 \%$ of the population, with tuberculosis in 5 patients. No injection-site reactions or pneumonia events were noted in the registry database. Treatment was discontinued in 12 out of 149 patients (8\%) owing to adverse events or a lack of efficacy. No new safety signals were observed when analyzing this registry data. The 
Table 2 Summary of disease outcome measures after 6 months of adalimumab biosimilar therapy in RA patients

\begin{tabular}{lcccc}
\hline Parameter & Baseline & At $\mathbf{6}$ months & \multicolumn{2}{l}{$\begin{array}{l}\text { *Change from baseline (mean } \pm \text { SD) } \\
{[\boldsymbol{n}=73]}\end{array}$} \\
\cline { 3 - 5 } & & & Change & \% Change \\
\hline DAS28-ESR & $7.10 \pm 0.43 ; 7.16(4.7,7.7)$ & $3.72 \pm 0.57 ; 2(2.72,5.71)$ & $-3.53 \pm 0.68$ & $-48.6 \pm 7.58^{*}$ \\
ESR & $100.3 \pm 12.36 ; 102.0(60,130)$ & $27.6 \pm 11.25 ; 26(10,72)$ & $-74.2 \pm 12.76$ & $-72.8 \pm 10.90^{*}$ \\
SJC & $11 \pm 2.7 ; 11.0(3,22)$ & $3 \pm 2.1 ; 3.0(8,16)$ & $-8 \pm 2.72$ & $-72.7 \pm 3.2^{*}$ \\
TJC & $13 \pm 3.3 ; 14.0(1.5,10)$ & $3 \pm 2.4 ; 3.0(6,21)$ & $-10 \pm 2.9$ & $-90.9 \pm 2.93^{*}$ \\
PGA & $7 \pm 1.4 ; 6.66(4,21)$ & $6 \pm 1.8 ; 6.9(1,18)$ & $-1 \pm 1.74$ & $-14.28 \pm 2.23^{*}$ \\
VAS-pain & $8.5 \pm 0.79 ; 9.0(5,10)$ & $2.5 \pm 0.91 ; 2(0,6)$ & $-6.2 \pm 1.09$ & $-71.4 \pm 11.00^{*}$ \\
\hline
\end{tabular}

Data are presented as mean \pm standard deviation or median (range)

DAS28-ESR Disease Activity Score-28 for Rheumatoid Arthritis with ESR, TJC total joint count, SJC single joint count, $P G A$ patient global assessment, $V A S$ visual analogue scale, $n$ number of patients with evaluable observations/data ${ }^{*} p<0.0001$; Wilcoxon signed rank test

Table 3 Summary of overall effectiveness and tolerability of adalimumab biosimilar in patients with RA at 6 months

\begin{tabular}{|c|c|c|c|c|}
\hline \multirow[t]{2}{*}{ Rating ${ }^{a}$} & \multicolumn{2}{|c|}{ Overall assessment of tolerability } & \multicolumn{2}{|c|}{ Overall assessment of efficacy } \\
\hline & $\begin{array}{l}\text { Physician's global } \\
\text { assessment } \\
n=126\end{array}$ & $\begin{array}{l}\text { Patient's global } \\
\text { assessment } \\
n=126\end{array}$ & $\begin{array}{l}\text { Physician's global } \\
\text { assessment } \\
n=126\end{array}$ & $\begin{array}{l}\text { Patient's global } \\
\text { assessment } \\
n=126\end{array}$ \\
\hline Excellent & $43(34 \%)$ & $85(67 \%)$ & $22(17 \%)$ & $50(40 \%)$ \\
\hline Good & $81(64 \%)$ & $37(29 \%)$ & $100(79 \%)$ & $72(57 \%)$ \\
\hline Fair & $2(2 \%)$ & $4(3 \%)$ & $4(3 \%)$ & $4(3 \%)$ \\
\hline Poor & 0 & 0 & 0 & 0 \\
\hline
\end{tabular}

Data presented as number of patients in the specified category (percentage)

$n$ total number of patients with evaluable observations/data

a 4 -point Likert Scale rating

global tolerability assessment ratings were good to excellent for $\geq 96 \%$ of patients.

\section{DISCUSSION}

Real-world evidence from the clinical use of high-end therapies, especially biosimilars, outside of clinical trials is required for the continuous appraisal of drug safety and efficacy in treated patients [11]. While most biosimilar products pass through rigorous analytical nonclinical and clinical comparisons with the originator before they are approved for use, they are still only similar to and not identical to or the same as the originator [17], and they are evaluated for approval differently by different authorities [18]. Hence, postmarketing surveillance studies or registries that monitor the realworld clinical use of an approved biosimilar provide additional validation of its effectiveness and safety $[8,11]$. Healthcare professionals usually have limited clinical information on a new product when they need to make an informed decision about whether to use that 
product. The availability of real-world data such as that examined in this report can help healthcare professionals to make a more informed and thus confident decision regarding biosimilar use and whether switching to a biosimilar from the originator is worthwhile, which could help to avoid the nocebo (negative placebo) effect $[8,18]$.

In our study, 24 weeks of bADA therapy led to significant improvements in the disease outcome measures, i.e. ESR, DAS-ESR and VAS-pain scores $(p<0.0001$ for all). About $48 \%, 48 \%$ and $34 \%$ of the patients achieved ACR20, ACR50, and ACR70 responses, respectively. The mean change in DAS28 at 24 weeks was - 3.53, and good and moderate EULAR responses were obtained in $15 \%$ and $58 \%$ of patients, respectively. Several pivotal clinical trials have demonstrated the efficacy of reference adalimumab in patients with RA, in terms of ACR criteria and the DAS-based EULAR response, when given subcutaneously every other week [3-5]. Patients with active RA who received adalimumab $40 \mathrm{mg}$ every other week in pivotal double-blind studies achieved ACR20 response rates of $63 \%$ and $65 \%$, ACR50 response rates of $52 \%$ and $39 \%$, and ACR70 response rates of $24 \%$ and $21 \%$ at 6 months [6]. The landmark ARMADA trial pointed to an ACR20 response in $67.2 \%$, an ACR50 response in $55.2 \%$ and an ACR70 response in $26.9 \%$ of RA patients receiving $40 \mathrm{mg}$ adalimumab for 24 weeks [3]. In another 26 -week phase III study, $46.0 \%$ of RA patients achieved ACR20, 22.1\% achieved ACR50 and $12.4 \%$ patients achieved ACR70 [4]. In a large, open-label trial in approximately 6000 RA patients previously treated with other anti-TNF agents, 12 weeks of adalimumab therapy was associated with ACR20 and ACR50 responses in about $60 \%$ and $33 \%$ of patients, respectively; $76 \%$ had a moderate and $23 \%$ had a good EULAR response. In addition, 12\% achieved DAS28 $<2.6$, indicating clinical remission [5]. A head-to-head clinical comparability trial of bADA versus reference adalimumab reported statistically similar response rates in both treatments at the end of 12 weeks: an ACR20 response in $82 \%$ vs. $79.2 \%$ patients, an ACR50 response in $46 \%$ vs. $43.4 \%$ patients, and an ACR70 response in $14 \%$ vs. $15.1 \%$ patients, respectively [13]. A report of the realworld use of bADA from a single Indian centre reported a reduction in DAS28 of $>1.2$ from baseline in $88 \%$ of the treated RA patients. At 6 months, the mean DAS28 score dropped to 3.32 from 6.26 at baseline. At 12 months, 58\% of the patients showed clinical remission or low disease activity [16].

Results from our analysis are in line with the published literature for adalimumab therapy discussed above. The slightly higher ACR50 and ACR70 response rates in our study as compared to the above reports may reflect only the good responders rather than the whole group. We also acknowledge that rates of ACR20 response and good EULAR response appear to be slightly lower than expected in these patients. However, the overall assessments pointed to good to excellent efficacy. The physician and patient global assessment accounting for efficacy outcomes (EULAR response) could have been subjective, i.e. a not so good improvement score may have been given by patients if they perceived a minor improvement in pain. On the other hand, the results of overall assessment (as presented in Table 3) were based on a 4-point Likert scale, wherein patients independently expressed that their overall confidence in the treatment was very high, which may reflect the suggested discrepancy.

It is important to note that only patients for whom complete data were available for 24 weeks were included in the analysis of efficacy outcomes. Hence, patients with delayed or missed follow-up details could have contributed to the lower overall values for ACR20 and EULAR good response. More importantly, this may be reflective of the prescription pattern and clinical management practices of Indian physicians for this patient pool. Biologics are employed more often as induction or debulking agents than as long-term disease-controlling therapies in clinical practice in India. Hence, biologics are initiated for a fixed duration, depending on how aggressively the treating physician approaches remission control, and are then discontinued or tapered off, only to be reintroduced for disease flares [19]. Hence, not all of the patients received therapy for all 6 months, while those who showed 
improvement during previous visits could have discontinued the therapy and would therefore not have been accounted for in 6-month outcome results. There is also a possibility that the cumulative good initial responses of patients who continued to receive the therapy may have led to the higher improvement scores (ACR50, moderate EULAR response) at 24 weeks. The prescription duration and the treatment approach of the physician are also often influenced more by the affordability of the treatment to the patient than the needs of the patient per se. Patient feedback about symptoms is an important influence on the usage of expensive therapies. Hence, in some cases there was an abrupt discontinuation of therapy, after which the patients were usually maintained on a conventional therapy while biologics were applied as rescue agents when there was a disease flare. Data from this registry are thus indicative of the clinical practice and usage patterns of the biosimilar in the real world in India.

The most common adverse events associated with adalimumab use are injection-site reactions, infections, and tuberculosis reactivation [6]. The safety profile of bADA was shown to be comparable to that of the originator and did not reveal any unexpected safety signals in the head-to-head comparison [13]. Real-world data from a single centre also did not yield any new findings relating to bADA use [14]. This registry has not prompted any unfavourable, unexpected safety concerns with the use of bADA in these patients. The reported side effects (fever, nausea, fungal infection, abdominal pain, headache, etc.) were in line with the established safety profile of adalimumab in RA patients. The low rates of injection-site reactions and adverse events may be attributable to the underreporting of adverse events owing to the non-randomized and well-informed patient pool, to missing data due to delayed or lost follow-up, to the open-label, observational, centre-specific data collection approach used by the registry, or to the succinate buffer base used in this biosimilar product [20]. This could also be reflective of the shorter and more varied durations of treatment (associated with real-world prescription patterns) analyzed in this study. In real world, often, patients with lesser risks are chosen for adalimumab use and hence, the safety data that has emerged out might have been limited. Nevertheless, most of the patients reported good to excellent tolerability overall.

Though limited by the real-world, open-label, non-interventional, observational nature of the study, registries like this offer an important monitoring tool for biosimilar treatments following regulatory approval. Data supporting the effectiveness and safety of biosimilars outside the controlled trial environment can help to reassure healthcare professionals of the dependability, interchangeability, and switchability of such products.

\section{CONCLUSION}

The post-marketing real-world clinical use of the adalimumab biosimilar Exemptia ${ }^{\mathrm{TM}}$ led to significant improvements in disease outcome scores, and the biosimilar showed good-to-excellent tolerability in Indian patients with RA. The results achieved with Exemptia ${ }^{\mathrm{TM}}$ were comparable to those attained with the reference adalimumab. Registries like the one used in the present work are important tools for probing the real-world prescription patterns and treatment strategies used in such patients from a country that has distinctive clinical practices.

\section{ACKNOWLEDGEMENTS}

Funding. This registry is maintained and sponsored by Cadila Healthcare Limited, India, as part of their post-marketing obligations in India. The study sponsor also funded the journal's article processing charges. All authors had full access to all of the data in this study and take complete responsibility for the integrity of the data and the accuracy of the data analysis.

Medical Writing, Editorial and Other Assistance. The authors would like to thank Dr. Ramnath Misra MD, FRCP, Head of the Department of Clinical Immunology and 
Rheumatology, SGPGIMS, Lucknow; Dr. Rohini Handa, Senior Consultant Rheumatologist at Apollo Indraprastha Hospitals, New Delhi; and Dr Debashish Danda MD, DM, FRCP, Founder and Professor Rheumatology, Christian Medical College, Vellore; India, for their expert review and guidance on the manuscript draft. The authors would also like to thank Dr. Sujata Sahwney, Ganga Ram Hospital, New Delhi; and Dr. Ashish Mathew, CMC Vellore, India, for their inputs and guidance for this paper. The authors would like to acknowledge the medical writing and editorial support provided by Dr. Hetal Shah, Founder \& Principal Consultant of $\mathrm{MeWriT}^{\mathrm{TM}}$, from Ahmedabad, India, in the preparation of this manuscript in collaboration with all the authors and reviewers, and in revising and styling the article as per journal requirements. Medical writing and editorial support was funded by Cadila Healthcare Limited, India. Data collection and analysis was outsourced to an independent clinical research organization, Karmic LifeSciences, India. The investigators were actively involved in the registry protocol, registry procedures, analysis, data reporting and the decision to publish this paper. Data analysis was funded by Cadila Healthcare Limited, India.

Authorship. All named authors meet the International Committee of Medical Journal Editors (ICMJE) criteria for authorship for this article, take responsibility for the integrity of the work as a whole, and have given their approval for this version to be published.

Disclosures. Mihir Gharia is a medical advisor at Cadila Healthcare Ltd., India. Dr. Sanjiv Kapoor, Dr. Vishwanath V Kaushik, Dr. Rahul Jain and Dr. Vijay Rao have nothing to disclose.

Compliance with Ethics Guidelines. Ethics committee approval (Intersystem Biomedica Ethics Committee, 09/02/2018, ref. ISBEC/NR3/DD-JJ/2018) was sought for the data analysis and publication. Patient consent for participation was duly taken. This study was conducted in accordance with the 1964 Declaration of Helsinki and applicable amendments.
Data Availability. The datasets obtained and/or analyzed during the current study are available from the corresponding author on reasonable request.

Open Access. This article is distributed under the terms of the Creative Commons Attribution-NonCommercial 4.0 International License (http://creativecommons.org/licenses/ by-nc/4.0/), which permits any noncommercial use, distribution, and reproduction in any medium, provided you give appropriate credit to the original author(s) and the source, provide a link to the Creative Commons license, and indicate if changes were made.

\section{REFERENCES}

1. Choy EH, Panayi GS. Cytokine pathways and joint inflammation in rheumatoid arthritis. $\mathrm{N}$ Engl J Med. 2001;344(12):907-16.

2. Rau R. Adalimumab (a fully human anti-tumour necrosis factor alpha monoclonal antibody) in the treatment of active rheumatoid arthritis: the initial results of five trials. Ann Rheum Dis. 2002;61(Suppl 2):ii70-3.

3. Weinblatt ME, Keystone EC, Furst DE, Moreland LW, Weisman MH, Birbara CA, et al. Adalimumab, a fully human anti-tumor necrosis factor alpha monoclonal antibody, for the treatment of rheumatoid arthritis in patients taking concomitant methotrexate: the ARMADA trial. Arthritis Rheum. 2003;48:35-45.

4. van de Putte LB, Atkins C, Malaise M, Sany J, Russell AS, van Riel PL, et al. Efficacy and safety of adalimumab as monotherapy in patients with rheumatoid arthritis for whom previous disease modifying antirheumatic drug treatment has failed. Ann Rheum Dis. 2004;63:508-16.

5. Bombardieri S, Ruiz AA, Fardellone P, Geusens P, McKenna F, Unnebrink $\mathrm{K}$, Research in Active Rheumatoid Arthritis (ReAct) Study Group, et al. Effectiveness of adalimumab for rheumatoid arthritis in patients with a history of TNF-antagonist therapy in clinical practice. Rheumatology (Oxford). 2007;6:1191-9.

6. Abbott Laboratories. Prescribing information for HUMIRA $^{\mathrm{TM}}$ (adalimumab). 2011. https://www. accessdata.fda.gov/drugsatfda_docs/label/2011/ 125057s0276lbl.pdf. Accessed 1 Jun 2018. 
7. Dörner T, Strand V, Cornes P, Gonçalves J, Gulácsi $\mathrm{L}$, Kay J, et al. The changing landscape of biosimilars in rheumatology. Ann Rheum Dis. 2016;75:974-82.

8. Rezk MF, Pieper B. Treatment outcomes with biosimilars: be aware of the nocebo effect. Rheumatol Ther. 2017;4:209-18.

9. Kaushik VV. Review of biosimilars of adalimumab. J Assoc Physicians India. 2017;65:15-21.

10. Business Standard B2B Bureau. Zydus Cadila launches biosimilar of adalimumab. Business Standard. 10 Dec 2014. http://www.business-standard.com/ content/b2b-pharma/zydus-cadila-launchesbiosimilar-of-adalimumab114121000039_1.html. Accessed 1 Jan 2019.

11. Declerck P, Farouk Rezk M. The road from development to approval: evaluating the body of evidence to confirm biosimilarity. Rheumatology (Oxford). 2017;56:iv4-13.

12. Bandyopadhyay S, Mahajan M, Mehta T, Singh AK, Parikh A, Gupta AK, et al. Physicochemical and functional characterization of a biosimilar adalimumab ZRC-3197. Biosimilars. 2015;5:1-18.

13. Jani RH, Gupta R, Bhatia G, Rathi G, Ashok Kumar $\mathrm{P}$, Sharma R, et al. A prospective, randomized, double-blind, multicentre, parallel-group, active controlled study to compare efficacy and safety of biosimilar adalimumab (Exemptia; ZRC-3197) and adalimumab (Humira) in patients with rheumatoid arthritis. Int J Rheum Dis. 2016;19:1157-68.
14. Mathew AJ, Ganapati A, Goel R, Danda D. Real-life safety profile of ZRC3197 (adalimumab biosimilar) in Indian patients with common rheumatic diseases. J Assoc Physicians India. 2017;65:30-2.

15. Kapoor S. Switching from other biologics to ZRC3197 (adalimumab biosimilar) in patients with spondyloarthropathy: a prospective evaluation from real-life. Clin Pract J Assoc Physicians India. 2017;65:26-9.

16. Sharma B. Clinical use of ZRC3197 (adalimumab biosimilar) in patients with inflammatory arthritis: a real-life experience. J Assoc Physicians. 2017;65:22-5.

17. Mysler E, Pineda C, Horiuchi T, Singh E, Mahgoub E, Coindreau J, Jacobs I. Clinical and regulatory perspectives on biosimilar therapies and intended copies of biologics in rheumatology. Rheumatol Int. 2016;36:613-25.

18. Markenson J, Alvarez DF, Jacobs I, Kirchhoff C. A practical guide about biosimilar data for health care providers treating inflammatory diseases. Biologics. 2017;11:13-21.

19. Shobha V, Rao V, Desai AM, Jois R, Srikantiah C, Dharmanand BG, et al. Prescribing patterns and safety of biologics in immune-mediated rheumatic diseases: Karnataka Biologics Cohort Study Group Experience. Ind J Rheumatol. 2019;14:17-20.

20. Gharia M, Sudhakar A. Reduced injection site pain with succinate buffer based adalimumab biosimilar (ZRC-3197) injection (SUFFER Study): An observational study. Ind J Rheumatol. 2019; in press. 